\title{
Bergman Kernel in Complex Analysis
}

\author{
Łukasz Kosiński and Włodzimierz Zwonek
}

\section{Contents}

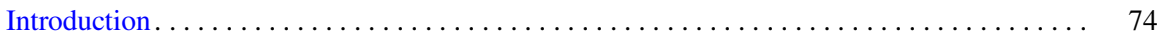

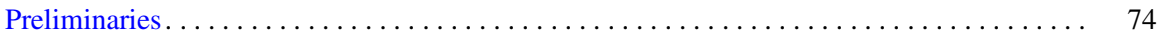

Some (Pluri)potential Theory and the Bergman Kernel in $\mathbb{C} \ldots \ldots \ldots \ldots \ldots \ldots \ldots \ldots \ldots$

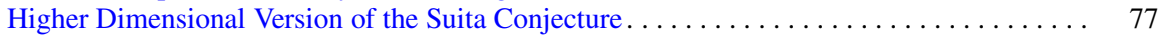

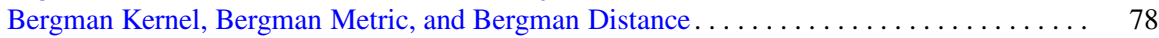

$\mathcal{L}_{\mathrm{h}}^{2}$-Domains of Holomorphy vs. Bergman Kernel. $\ldots \ldots \ldots \ldots \ldots \ldots \ldots \ldots \ldots \ldots \ldots$

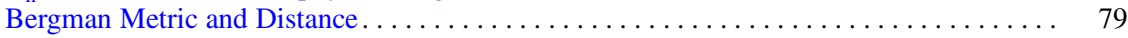

Bergman Kernel Function vs. Biholomorphic and Proper Holomorphic Mappings . . . . . . . 80

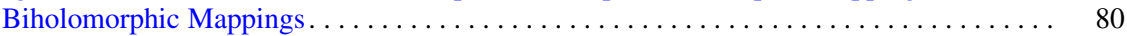

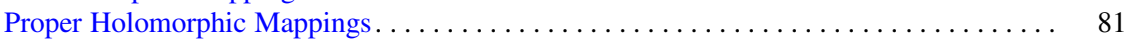

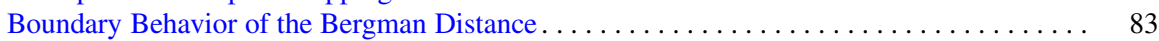

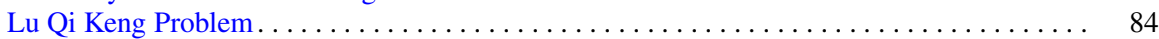

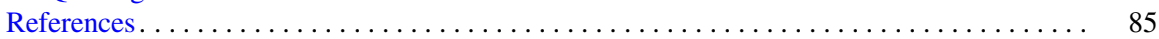

\begin{abstract}
In this survey a brief review of results on the Bergman kernel and Bergman distance concentrating on those fields of complex analysis which remain in the focus of the research interest of the authors is presented. The topics discussed contain general discussion of $\mathcal{L}_{\mathrm{h}}^{2}$ spaces, behavior of the Bergman distance, regularity of extension of proper holomorphic mappings, and recent development in the theory of Bergman distance stemming from the pluripotential theory and very short discussion of the Lu Qi Keng problem.
\end{abstract}

Ł. Kosiński • W. Zwonek ( $₫)$

Faculty of Mathematics and Computer Science, Department of Mathematics, Jagiellonian University, Kraków, Poland

e-mail: lukasz.kosinski@im.uj.edu.pl; wlodzimierz.zwonek@im.uj.edu.pl 


\section{Introduction}

In the paper only the case of domains $D \subset \mathbb{C}^{n}$ is considered. Denote

$$
\mathcal{L}_{\mathrm{h}}^{2}(D):=\mathcal{L}^{2}(D) \cap \mathcal{O}(D)
$$

- the space of square integrable holomorphic functions on $D$. The problems discussed below will often require the additional assumption of boundedness of the domain $D$. However, some of the topics will be interesting in the case of unbounded $D$. Therefore, although it will make the presentation sometimes more complicated, we leave the definition in the case of general domains $D$ imposing additional condition when needed.

$\mathcal{L}_{\mathrm{h}}^{2}(D)$ is a closed subspace of $\mathcal{L}^{2}(D)$. It follows from the Cauchy formula that the $\mathcal{L}^{2}$-convergence of elements in $\mathcal{L}_{\mathrm{h}}^{2}(D)$ implies the local uniform convergence. Let $K_{D}$ denote the reproducing kernel of the Hilbert space $\mathcal{L}_{\mathrm{h}}^{2}(D)$ which means, in particular, that for any $z \in D$ and $f \in \mathcal{L}_{\mathrm{h}}^{2}(D)$, one gets the equality ( $\lambda^{2 n}$ denotes the $2 n$-dimensional Lebesgue measure)

$$
f(z)=\int_{D} f(w) \overline{K_{D}(w, z)} d \lambda^{2 n}(w) .
$$

Let $P_{D}$ denote the orthogonal projection $\mathcal{L}^{2}(D) \rightarrow \mathcal{L}_{\mathrm{h}}^{2}(D)$. The operator $P_{D}$ is called the Bergman projection. A generalization to suitable weighted $\mathcal{L}_{\mathrm{h}}^{2}$-spaces is also possible. But below only the special case presented above is considered.

The recent survey of the theory of Bergman functions - writing Bergman function we mean one of three discussed objects: Bergman kernel, Bergman metric, or Bergman distance (the latter two to be defined later) - especially with the stress put on its links with the pluripotential theory, may be found in $[8,11]$, and [28]. The topics contained below reflect the interest of the authors and refer to different aspects of the interactions of the theory of Bergman functions and classical complex analysis. The authors are also aware that the list of references is very much incomplete but at the moment the literature on the Bergman functions in complex analysis is so wide that it would not be possible to contain all the references and therefore a very subjective choice was made by the authors.

\section{Preliminaries}

A very important tool in complex analysis is pluripotential theory which is based on the properties of plurisubharmonic functions. Recall that an upper semicontinuous function $u: D \rightarrow[-\infty, \infty)$ (possibly equal identically to $-\infty$ ) is called plurisubharmonic if $u$ restricted to $l \cap D$ is subharmonic (possibly equal identically to $-\infty$ on connected components of $l \cap D$ ) for any affine complex line $l$ intersecting $D$. 
A set $P$ is called (pluri)polar if $P \subset u^{-1}(-\infty)$ for some (pluri)subharmonic $u$ defined on $\mathbb{C}^{n}$ where $u$ is not identically $-\infty$ (or equivalently the property is given locally - use the Josefson theorem - see [29]).

A domain $D \subset \mathbb{C}^{n}$ is called pseudoconvex if there is a plurisubharmonic function $u$ defined on $D$ such that $u$ is exhaustive, i.e., for any sequence $\left(z^{k}\right) \subset D$ without accumulation points in $D$ we have $\lim _{k \rightarrow \infty} u\left(z^{k}\right)=\infty$.

A Liouville-type theorem states that $\mathcal{L}_{\mathrm{h}}^{2}\left(\mathbb{C}^{n}\right)=\{0\}$. On the other hand in the case when $D$ is bounded, we get that $\operatorname{dim} \mathcal{L}_{\mathrm{h}}^{2}(D)=\infty$ (all the polynomials are in $\mathcal{L}_{\mathrm{h}}^{2}(D)$ ). It is interesting that these two extremal cases are in some sense generic at least for $n=1$. One has, namely, the following result (see, e.g., [48]):

Theorem 1. Let $D$ be a domain in $\mathbb{C}$. Then the following are equivalent:

- $\mathcal{L}_{\mathrm{h}}^{2}(D) \neq\{0\}$

- $\operatorname{dim} \mathcal{L}_{\mathrm{h}}^{2}(D)=\infty$,

- $D$ is not of the form $\mathbb{C} \backslash P$ for any closed polar set $P \subset \mathbb{C}$,

- the system of functions $\left\{K_{D}(\cdot, z): z \in D\right\}$ is linearly independent,

- $K_{D}(z, z)>0$ for any $z \in D$.

In higher dimensions the situation is totally different though not fully understood. For instance, $\mathcal{L}_{\mathrm{h}}^{2}(\mathbb{D} \times \mathbb{C})=\{0\}$ (here and in the sequel $\mathbb{D}$ denotes the unit disk in C). Moreover, for any $k \in \mathbb{N}$ there exists an unbounded Reinhardt domain $D_{k}$ in $\mathbb{C}^{2}$ such that $\operatorname{dim} \mathcal{L}_{\mathrm{h}}^{2}\left(D_{k}\right)=k$ (see [48]). Note that, however, the domains $D_{k}$ constructed in [48] are not pseudoconvex. It is still an open problem whether there exists a pseudoconvex domain $D$ such that $0<\operatorname{dim} \mathcal{L}_{\mathrm{h}}^{2}(D)<\infty$. Only partial results in this direction are known - see, e.g., [31].

It is well known that having given an orthonormal basis $\left\{\varphi_{k}\right\}_{k \in J}$ of the space $\mathcal{L}_{\mathrm{h}}^{2}(D)$ ( $J$ is at most countable), one has the following equality:

$$
K_{D}(z, w)=\sum_{k \in J} \varphi_{k}(z) \overline{\varphi_{k}(w)},
$$

called the Zaremba decomposition (cf. [47]). Denote the Bergman kernel on the diagonal by $k_{D}(z):=K_{D}(z, z) \geq 0, z \in D$.

The convergence in (4.3) is, in particular, absolute and locally uniform. The Hartogs theorem on separate analyticity allows to conclude that $K_{D}$ is real analytic.

Recall some basic transformation formulas for the Bergman kernel.

Let $F: D \rightarrow G$ be a biholomorphic mapping between domains $D, G \subset \mathbb{C}^{n}$. Then

$$
K_{G}(F(w), F(z)) \operatorname{det} F^{\prime}(w) \overline{\operatorname{det} F^{\prime}(z)}=K_{D}(w, z), w, z \in D .
$$

For domains $D \subset \mathbb{C}^{n}, G \subset \mathbb{C}^{m}$ we have the property

$$
K_{D \times G}\left(\left(w_{1}, w_{2}\right),\left(z_{1}, z_{2}\right)\right)=K_{D}\left(w_{1}, z_{1}\right) K_{G}\left(w_{2}, z_{2}\right),
$$

where $\left(w_{1}, w_{2}\right),\left(z_{1}, z_{2}\right) \in D \times G$. 
For a balanced pseudoconvex domain $D$ in $\mathbb{C}^{n}$ it follows from the Taylor expansion of functions in $\mathcal{L}_{\mathrm{h}}^{2}(D)$ that there is an orthonormal basis of $\mathcal{L}_{\mathrm{h}}^{2}(D)$ consisting of homogeneous polynomials. Consequently, thanks to (4.3), one has the following formula $K_{D}(0, z)=1 / \lambda^{2 n}(D), z \in D$. This implies, because of (4.4), a complete effective formula in all balanced domains with transitive automorphism group. In particular, one gets the equalities $\left(\mathbb{B}_{n}\right.$ denotes the unit Euclidean ball in $\left.\mathbb{C}^{n}\right)$

$$
\begin{aligned}
& K_{\mathbb{D}^{n}}(w, z)=\frac{1}{\pi^{n}} \prod_{j=1}^{n} \frac{1}{\left(1-z_{j} \bar{w}_{j}\right)^{2}}, \\
& K_{\mathbb{B}_{n}}(w, z)=\frac{n !}{\pi^{n}} \frac{1}{(1-\langle w, z\rangle)^{n+1}} .
\end{aligned}
$$

Example 1. Consider the domain

$$
D_{\alpha}:=\left\{\left(z_{1}, z_{2}\right) \in \mathbb{C}^{2}:\left|z_{2}\right|<1,\left|z_{2}\right|\left|z_{1}\right|^{\alpha}<1\right\} .
$$

Then the function $z_{1}$ belongs to $\mathcal{L}_{\mathrm{h}}^{2}\left(D_{\alpha}\right)$ for any $\alpha>2$. This implies that $K_{D_{\alpha}} \not \equiv 0$, whereas $K_{D_{\alpha}}(z, 0)=0$ which shows that there is a dramatic difference between the theory of the Bergman kernel in one-dimensional and higher-dimensional case (compare Theorem 1).

\section{Some (Pluri)potential Theory and the Bergman Kernel in $\mathbb{C}$}

There is a very clear interaction between the Bergman kernel and (pluri)potential theory. Below classical results will be presented. Some of them have generalizations in higher dimensions. Part of the presented results has been stimulating the recent developments in the theory of the Bergman kernel. The good reference for the pluripotential theory used below is monograph [33].

First recall the notion of the pluricomplex Green function with the logarithmic pole at $p \in D$ where $D \subset \mathbb{C}^{n}$. Define (see [32])

$$
g_{D}(p, z):=\sup \{u(z)\},
$$

where the supremum is taken over all plurisubharmonic $u: D \rightarrow[-\infty, 0)$ such that $u(\cdot)-\log \|\cdot-p\|$ is bounded above near $p$. In the case $n=1$ the function is the classical Green function (up to a sign). Recall the formula (for $D \subset \mathbb{C}$ ) admitting the nontrivial Green function $\left(g_{D}(w, z)>-\infty, w \neq z\right.$ or such that $\mathbb{C} \backslash D$ is not polar - see, e.g., [21] or [42])

$$
K_{D}(z, w)=\frac{2}{\pi} \frac{\partial^{2} g_{D}}{\partial z \partial \bar{w}}(z, w), z \neq w .
$$

One may also show for $D$ as above that 


$$
K_{D}(z, z)=\frac{1}{\pi} \frac{\partial^{2}}{\partial z \partial \bar{z}}\left(\log c_{D}(z)\right),
$$

where $c_{D}(z):=\exp \left(\lim _{w \rightarrow z}\left(g_{D}(z, w)-\log |w-z|\right)\right), z \in D$, is the logarithmic capacity of the complement of $D$ with respect to $z$. In [45] the author, motivated by the upper estimate for the curvature of the metric induced by $c_{D}$, conjectured that

$$
c_{D}^{2}(z) \leq \pi K_{D}(z, z) \text { for any } z \in D .
$$

In fact, Suita showed the equality in the case of the disk (it is trivial) and obtained a strict inequality when $D$ is an annulus. There was no progress with the above conjecture till T. Ohsawa attacked the problem with methods of higher-dimensional complex analysis. In fact he observed that the Suita conjecture may be expressed as a problem of extension of square integrable holomorphic functions (see [38]). Since that time a decisive progress has been made. Namely, it was shown that the estimate $c_{D}^{2}(z) \leq C \pi K_{D}(z, z)$ holds with some constant $C$. Making use of the ideas of Ohsawa-Takegoshi extension theorem (see [39]), Ohsawa showed the estimate with $C=750$ (see [38]). Then in a series of subsequent papers, the estimate was being improved. Finally the optimal constant, and thus the proof of the Suita conjecture, was presented by Z. Błocki in [9].

\section{Higher Dimensional Version of the Suita Conjecture}

In the proof of the Suita conjecture, an estimate for $\mathcal{L}_{\mathrm{h}}^{2}$-functions in pseudoconvex domains (in higher dimension!) was used.

In the paper [10] the idea of the proof of the Suita conjecture was reduced to the following theorem which was also a generalization of similar earlier results (see, e.g., [26]):

Theorem 2 (see [10]). Let $D$ be a bounded pseudoconvex domain in $\mathbb{C}^{n}, f \in$ $\mathcal{L}_{\mathrm{h}}^{2}(D), z \in D, a \geq 0$. Then

$$
\frac{|f(z)|^{2}}{k_{D}(z)} \leq e^{2 n a} \int_{\left.\left\{g_{D}(z,)\right)<-a\right\}}|f|^{2} d \lambda^{2 n} .
$$

Passing with $a \rightarrow \infty$ for $f \equiv 1$ and making use of some standard approximation process, one gets the following version of the multidimensional Suita conjecture (see [13]).

Theorem 3. Let $D$ be a bounded pseudoconvex domain in $\mathbb{C}^{n}, z \in D$. Then

$$
k_{D}(z) \geq \frac{1}{\lambda^{2 n}\left(I_{D}(z)\right)},
$$


where $I_{D}(z):=\left\{X \in \mathbb{C}^{n}: A_{D}(z ; X)<1\right\}$ is the Azukawa indicatrix at $z \in D$.

Recall that the Azukawa pseudometric is defined as follows

$$
A_{D}(z ; X):=\exp \left(\limsup _{\lambda \rightarrow 0}\left(g_{D}(z, z+\lambda X)-\log |\lambda|\right)\right),(z ; X) \in D \times \mathbb{C}^{n} .
$$

It is obvious that $c_{D}(z)=A_{D}(z ; 1), z \in D \subset \mathbb{C}$. Moreover, the upper limit above becomes the limit in many cases, for instance, if $D$ is hyperconvex (see [49]). When $D$ is convex, the Lempert theorem implies that the Azukawa pseudometric coincides with the Kobayashi one (see [36]).

The above theorem may be expressed by the following inequality

$$
F_{D}(z):=\left(k_{D}(z) \lambda^{2 n}\left(I_{D}(z)\right)\right)^{1 / n} \geq 1 \text {. }
$$

The function $F_{D}$ is a biholomorphic invariant. Moreover, in the case when $D$ is convex, we have the inequality $F_{D} \leq 4$ [13].

\section{Bergman Kernel, Bergman Metric, and Bergman Distance}

When restricted to the diagonal, another presentation of the Bergman kernel is possible

$$
k_{D}(z)=\sum_{k \in J}\left|\varphi_{k}(z)\right|^{2}=\sup \left\{|f(z)|^{2}: f \in \mathcal{L}_{\mathrm{h}}^{2}(D),\|f\| \leq 1\right\} .
$$

It follows from (4.17) that for any two domains $D \subset G \subset \mathbb{C}^{n}$, the following inequality $k_{D}(z) \geq k_{G}(z), z \in D$ holds.

The above description of the Bergman kernel on the diagonal implies that the function

$$
D \ni z \mapsto \log k_{D}(z)
$$

is plurisubharmonic.

\section{$\mathcal{L}_{\mathrm{h}}^{2}$-Domains of Holomorphy vs. Bergman Kernel}

A consequence of the Riemann-type removability theorem is that the Bergman kernel extends through analytic sets. Therefore, it is easy to find pseudoconvex domains with the Bergman kernel (restricted to the diagonal) not being exhaustive (take the punctured unit disc $\mathbb{D}_{*}$ ). However, there is a nice description of $\mathcal{L}_{\mathrm{h}}^{2}$ domains of holomorphy expressed with the aid of the Bergman kernel. 
Recall that a domain $D \subset \mathbb{C}^{n}$ is called an $\mathcal{L}_{\mathrm{h}}^{2}$-domain of holomorphy if for any pair of open sets $U_{1}, U_{2} \subset \mathbb{C}^{n}$ with $\emptyset \neq U_{1} \subset D \cap U_{2} \neq U_{2}, U_{2}$ connected, there is an $f \in \mathcal{L}_{\mathrm{h}}^{2}(D)$ such that for any $F \in \mathcal{O}\left(U_{2}\right)$ we have $f_{\mid U_{1}} \neq F_{\mid U_{1}}$ (see, e.g., [27]).

Theorem 4 (see [40]). Let $D$ be a pseudoconvex bounded domain in $\mathbb{C}^{n}$. Then the following are equivalent:

- $\quad D$ is an $\mathcal{L}_{\mathrm{h}}^{2}$-domain of holomorphy,

- $\lim \sup _{z \rightarrow \partial D} k_{D}(z)=\infty$,

- For any $z \in \partial D$ and any open neighborhood $U$ of $z$, the set $U \cap \partial D$ is not pluripolar.

\section{Bergman Metric and Distance}

Under the condition $k_{D}(z)>0$ (e.g., when $D$ is bounded), the smoothness and plurisubharmonicity of $\log k_{D}(z)$ assures the nonnegativity of the Levi form of $\log k_{D}$. This leads to the definition of the Bergman pseudometric as follows:

$$
\beta_{D}(z ; X):=\sqrt{\sum_{j, k=1}^{n} \frac{\partial^{2} \log k_{D}(z)}{\partial z_{j} \bar{\partial} z_{k}} X_{j} \overline{X_{k}}},(z ; X) \in D \times \mathbb{C}^{n}
$$

When $D$ is bounded the function $\beta_{D}$ is strictly positive definite. In such a case one defines the Bergman distance as follows:

$$
b_{D}(w, z):=\inf \left\{\int_{0}^{1} \beta_{D}\left(\alpha(t) ; \alpha^{\prime}(t)\right) d t\right\},
$$

where the infimum is taken over all $\alpha:[0,1] \rightarrow D$ piecewise $C^{1}, \alpha(0)=w$, $\alpha(1)=z$.

The transformation formula for the Bergman kernel easily implies that for a biholomorphic mapping $F: D \rightarrow G$, one has the equalities

$$
\begin{gathered}
\beta_{G}\left(F(z) ; F^{\prime}(z) X\right)=\beta_{D}(z ; X),(z ; X) \in D \times \mathbb{C}^{n}, \\
b_{G}(F(w), F(Z))=b_{D}(w, z),(w, z) \in D \times D .
\end{gathered}
$$

Recall that the above functions do not decrease (in general) under inclusion of sets (see [6] and [41] for a one-dimensional example).

The boundary behavior of the Bergman kernel, metric, and distance is of great importance in the theory of complex analysis that will be presented in subsequent sections. 


\section{Bergman Kernel Function vs. Biholomorphic and Proper Holomorphic Mappings}

\section{Biholomorphic Mappings}

The Bergman projection operator plays the fundamental role in the boundary behavior of biholomorphic mappings. Generally speaking, if a biholomorphic mapping $F: D \rightarrow G$, where $D$ and $G$ are bounded domains in $\mathbb{C}^{n}$, extends to a diffeomorphism between $\bar{D}$ and $\bar{G}$ (or extends to a biholomorphism between neighborhoods of $\bar{D}, \bar{G}$ ), then certain geometric properties of the topological boundaries of $D$ and $G$ are preserved by $f$.

The most important result in this direction is the theorem of Fefferman which states

Theorem 5 (see [24]). Let $D$ and $G$ be $\mathcal{C}^{\infty}$-smoothly bounded strictly pseudoconvex domains in $\mathbb{C}^{n}$. Then any biholomorphic mapping $F: D \rightarrow G$ extends to a $\mathcal{C}^{\infty}$ diffeomorphism from $\bar{D}$ onto $\bar{G}$.

Before the above theorem was proven, N. Vormoor had showed that such a mapping $F$ extends continuously up to the boundary. To get the differentiability, Fefferman involved machinery related to the Bergman metric. More precisely, the starting point was the following basic fact: $F$ is an isometry when both domains are equipped with their Bergman metrics. Then, differential geometric calculations on the behavior of geodesic near the boundary which were possible to perform by obtaining a very deep and precision description of the Bergman kernel near the diagonal allowed Fefferman to show that any isometry between Bergman metric of $D$ and $G$ is smooth up to the boundary of domains.

In one complex variable a link between the Bergman kernel and boundary properties of biholomorphic mappings had a long history and was intensively studied. In several complex variables, such a connection was established by already presented Fefferman's methods, and this relationship became closer and intimate, thanks to Bell and Ligocka works. The most remarkable paper that should be mentioned here is [5], where Fefferman's theorem was extended by Bell and Ligocka (among others to pairs of weakly pseudoconvex domains with real analytic boundaries) and its proof was simplified. Thus, it showed that Theorem 5 was a rather simple consequence of subelliptic estimates for $\bar{\partial}$.

Recall here that a domain $D$ is said to satisfy condition $(R)$ if it is smooth and for each $j \geq 0$ there is $m>0$ such that the Bergman projection for $D$ is a bounded operator from $W^{j+m}(D)$ to $W^{j}(D)$. Here $W^{k}(D)$ denotes the usual Sobolev space of function on $D$ with distributional derivatives up to order $k$ in $\mathcal{L}^{2}(D)$. It may be shown that condition $(\mathrm{R})$ is equivalent to the fact that the Bergman projection $P_{D}$ maps $\mathcal{C}^{\infty}(\bar{D})$ into $\mathcal{C}^{\infty}(\bar{D})$.

It is a deep fact that condition (R) holds on smooth strictly pseudoconvex domains. It is also satisfied for some weakly pseudoconvex domains, for instance, it holds for a class of bounded pseudoconvex domains with real analytic boundaries. 
Generally, subelliptic estimates for $\bar{\partial}$-Neumann operator on the domain $D$ imply condition $(\mathrm{R})$ but $(\mathrm{R})$ is weaker.

Theorem 6 ([5]). If $D$ and $G$ are smoothly bounded domains satisfying condition $(R)$, then any biholomorphic mapping extends smoothly up to the boundary.

The above result was improved by Bell (see [1]) by showing that it suffices for just one of the domains to satisfy condition (R). The relationship between the Bergman kernel, condition (R), geometry of domains, and their group of automorphisms has been intensively studied since then (e.g., $[17,25])$.

\section{Proper Holomorphic Mappings}

It is hard to apply Fefferman's methods to proper holomorphic mappings. Recall that a mapping $F$ between domains $D$ and $G$ of $\mathbb{C}^{n}$ is called proper if preimages of compact subsets of $G$ are compact in $D$. It is known that any proper and holomorphic mapping $F: D \rightarrow G$ is open and that $\Sigma:=F\left(\left\{z \in D: \operatorname{det} F^{\prime}(z)=\right.\right.$ $0\})$ is analytic. Moreover, $F$ restricted to $D \backslash F^{-1}(\Sigma)$ is a finite-branched covering onto $G \backslash \Sigma$. In particular, $F$ has finitely many holomorphic inverses defined locally on $G \backslash \Sigma$.

A connection between the Bergman kernel and proper holomorphic mappings was found in Bell's papers (see [2] and [3]). The following two transformation rules that are of the key importance are worth mentioning.

The Bergman projections for $D$ and $G$ transform under $F$ according to the formula

$$
P_{G}\left(\operatorname{det} F^{\prime} \cdot h \circ F\right)=\operatorname{det} F^{\prime} \cdot\left(P_{D} h\right) \circ F, \quad h \in \mathcal{L}^{2}(D) .
$$

Roughly speaking, the above formula says that the Bergman projections transform under proper holomorphic mappings as under biholomorphic mappings. Quite a direct consequence of (4.23) is the following rule for the Bergman kernel (a generalization of (4.4)):

Theorem 7. Let $F: D \rightarrow G$ be proper holomorphic mapping between domains of $\mathbb{C}^{n}$. Let $\Phi_{1}, \ldots, \Phi_{m}$ be local inverses to $F$ defined locally in $G \backslash \Sigma$. Then the Bergman kernel transforms by the formula

$$
\sum_{j=1}^{m} K_{G}\left(z, \Phi_{k}(w)\right) \overline{\operatorname{det} \Phi_{k}^{\prime}(w)}=\operatorname{det} F^{\prime}(z) K_{D}(F(z), w),
$$

where $(z, w) \in D \times(G \backslash \Sigma)$. 
In [4] and independently in [22], extension of results obtained in [5] and [1] for biholomorphisms to proper holomorphic mappings was given.

It was an open problem for a long time whether condition $(\mathrm{R})$ is satisfied by all smooth and bounded pseudoconvex domains. The answer turned out to be negative the famous Diederich-Fornaess worm domain (see [22]) serves as a counterexample (see [20]). Thus the methods developed by Bell, Ligocka, Diederich, Fornaess, Catlin, et al. cannot work in the general case.

Nevertheless, there are methods exploiting the Bergman kernel which work for classes of domains possessing some actions playing on them (see [3]).

A Reinhardt domain in $\mathbb{C}^{n}$ is a domain $D$ such that

$$
\left(e^{i \theta_{1}} z_{1}, \ldots, e^{i \theta_{n}} z_{n}\right) \in D
$$

for any $z=\left(z_{1}, \ldots, z_{n}\right) \in D$ and real $\theta_{1}, \ldots, \theta_{n}$. If we assume that (4.24) holds only for real $\theta_{j}$ such that $\theta_{1}=\cdots=\theta_{n}$, the domain $D$ is said to be circular, which means that balanced domain is circular. There is a very important reason for studying these kinds of domains; namely, logarithmically convex Reinhardt domains are the domains of convergence of power series. Moreover, a number of domains appearing naturally or serving as important examples possess some actions acting on them and their geometry; in particular, their holomorphic equivalence attracts a lot of attention.

The most general result in this direction is the following (see [2]):

Theorem 8. Let $D$ and $G$ be bounded domains of $\mathbb{C}^{n}$. Suppose that $G$ is circular and contains the origin. Assume, moreover, that the Bergman kernel function of the domain $D$ satisfies the following property: for any open and relatively compact subset $K$ of $D$, there is an open neighborhood $U$ of $\bar{D}$ such that $(z, w) \mapsto K_{D}(z, \bar{w})$ extends holomorphically to a neighborhood $U \times \tilde{K}$, where $\tilde{K}=\{\bar{x}: x \in K\}$. Then any proper holomorphic mapping $f: D \rightarrow G$ extends holomorphically to $a$ neighborhood of $\bar{D}$.

The assumption on the Bergman kernel is satisfied in a wide class of domains. It contains among others bounded domains that are complete circular with respect to the origin and whose Minkowski functionals are continuous (recall that there are quite "wild" balanced pseudoconvex domains without continuous Minkowski functionals).

As already mentioned, if a biholomorphic mapping of a domain $D$ onto a domain $G$ extends holomorphically to a neighborhood of $\bar{D}$, the number of invariants of topological boundaries of the domains must be preserved. A simple and very elementary example that may serve here as an illustration of application of these ideas is the biholomorphic inequivalence of the polydisk and the Euclidean ball (in dimension at least two) - if there existed a biholomorphic mapping between them, it would extend to a biholomorphic mapping between neighborhoods of their closures which is impossible as smoothness of boundaries is not preserved. 
Using Theorem 8 and more intimate relationship between the Bergman kernel and proper holomorphic mappings, one may get (see $[2,3])$ the following result: Suppose that $F: D \rightarrow G$ is a proper holomorphic mapping between bounded complete circular domains containing the origin such that $F^{-1}(0)=\{0\}$, then $F$ is a polynomial mapping.

There are a number of generalizations and extensions of the above results (see, e.g., [35]). However, roughly speaking, the ideas of Bell, Fefferman, and Ligocka are the starting point for almost all of them.

\section{Boundary Behavior of the Bergman Distance}

An important problem of the Bergman functions is the boundary behavior of the Bergman distance or, in some cases, the problem of Bergman completeness, i.e., the study of a situation when the metric space $\left(D, b_{D}\right)$ is complete.

First, make some immediate remarks. A necessary property for a bounded domain to be Bergman complete is its pseudoconvexity - this was observed in [34]. An example of the punctured disk shows that the converse does not hold in general. The most important tool which is used in (almost) all proofs of Bergman completeness is the Kobayashi criterion (see [34]).

Theorem 9. Let $D$ be a bounded domain in $\mathbb{C}^{n}$. Assume that for any $f \in \mathcal{L}_{\mathrm{h}}^{2}(D)$, the following equality holds

$$
\lim _{z \rightarrow \partial D} \frac{|f(z)|^{2}}{k_{D}(z)}=0
$$

Then $D$ is Bergman complete.

There are some formally stronger versions of the above result (see [8]). Recall that the converse implication does not hold, even in the case $n=1$ (see [50] and also [30, 51]).

The most general and the most important class of domains that turned out to be Bergman complete consists of hyperconvex domains. Recall that a bounded domain $D \subset \mathbb{C}^{n}$ is called hyperconvex if there is a negative plurisubharmonic exhaustive function $u: D \rightarrow[-\infty, 0)$ (negative exhaustive means: $\lim _{z \rightarrow \partial D} u(z)=0$ ). Equivalently, the pluricomplex Green function for some (equivalently, any) pole $p \in D$ is exhaustive.

Stimulated by the ideas developed in [18], one may prove the following.

Theorem 10 (see $[12,26])$. Let $D$ be a hyperconvex domain in $\mathbb{C}^{n}$. Then $D$ is Bergman complete. 
The proof of the above theorem is based on the connection between the Bergman kernel, the pluricomplex Green function, and the Kobayashi criterion. More precisely, making use of the inequality (4.13) (with $a=1$ ), we get

$$
\frac{|f(z)|^{2}}{k_{D}(z)} \leq e^{2 n} \int_{\left\{g_{D}(\cdot, z)<-1\right\}}|f|^{2} d \lambda^{2 n}, f \in \mathcal{L}_{\mathrm{h}}^{2}(D),
$$

which holds for any pseudoconvex bounded domain $D$. And now the convergence $\lim _{z \rightarrow \partial D} \lambda^{2 n}\left(\left\{g_{D}(\cdot, z)<-1\right\}\right)=0$, which holds for hyperconvex domains, together with the Kobayashi criterion completes the proof.

There are (even in dimension one) non-hyperconvex Bergman complete domains (see [19]).

Note that the Kobayashi criterion does not give any quantitative estimate for the Bergman distance near the boundary. However, the following estimate was shown in [23].

Theorem 11. Let $D$ be a pseudoconvex domain with $C^{2}$-boundary. Then the following estimate holds $\left(d_{D}(w)\right.$ denotes the distance of $w$ to the boundary of $\left.D\right)$ :

$$
b_{D}(z, w) \geq C \log \left(\log \left(1 / d_{D}(w)\right)\right)
$$

for some positive constant $C$, fixed $z \in D$ and $w$ close to $\partial D$.

Diederich and Ohsawa formulated the conjecture, still open, that the righthand side of inequality (4.27) may be replaced by $C \log \left(1 / d_{D}(w)\right)$ (which is best possible as the example of strongly pseudoconvex domains shows). The best lower estimate so far is the one obtained in [7] which gives the behavior like $\log \left(1 / d_{D}(w)\right) / \log \left(\log \left(1 / d_{D}\right)\right)$. In the proofs of the results mentioned once more the pluripotential theory is used.

\section{Lu Qi Keng Problem}

The Lu Qi Keng problem is to determine domains $D \subset \mathbb{C}^{n}$ for which the Bergman kernel has no zeros (see [37]). The motivation for considering that problem was the idea of representative domain, a notion introduced by S. Bergman, to which a given domain may be mapped by the representative coordinates. Representative coordinates are given locally with the help of derivatives of the logarithm of the Bergman kernel. It is therefore clear that zeroes of the Bergman kernel make the obstruction for the global definition.

In dimension one it is known that an annulus, or generally any finitely connected but not simply connected smooth domain is not a Lu Qi Keng domain (see [43, 46]). It was believed for some time that in higher dimension any pseudoconvex, topologically trivial domain was a Lu Qi Keng. However, the counterexample was 
found (see [44] and [14]). Later it turned out that "most" of bounded pseudoconvex domains are not Lu Qi Keng in the sense that the class of domains not being Lu Qi Keng forms a nowhere dense set in a suitable topology.

Since that time a number of effective examples of domains being Lu Qi Keng (or the opposite) were found (for the list of references, see, e.g., [15] or [28]). In the construction of domains being not Lu Qi Keng, several techniques were developed: deflation, use of the transformation formula for proper holomorphic mappings, and others (see, e.g., [16]).

\section{References}

1. Bell, S.: Biholomorphic mappings and the $\bar{\partial}$-problem. Ann. Math. (2) 114(1), 103-113 (1981)

2. Bell, S.: Proper holomorphic mappings between circular domains. Comment. Math. Helv. 57(4), 532-538 (1982)

3. Bell, S.: The Bergman kernel function and proper holomorphic mappings. Trans. Am. Math. Soc. 270(2), 685-691 (1982)

4. Bell, S., Catlin, D.: Boundary regularity of proper holomorphic mappings. Duke Math. J. 49(2), 385-396 (1982)

5. Bell, S., Ligocka, E.: A simplification and extension of Fefferman's theorem on biholomorphic mappings. Invent. Math. 57(3), 283-289 (1980)

6. Bergman, S.: Zur Theorie von pseudokonformen Abbildungen. Mat. Sb. 43, 79-96 (1936)

7. Błocki, Z.: The Bergman metric and the pluricomplex Green function. Trans. Am. Math. Soc. 357(7), 2613-2625 (2005)

8. Błocki, Z.: The Bergman kernel and pluripotential theory. In: Potential theory in Matsue, 1-9. Advanced Studies in Pure Mathematics, vol. 44, Mathematics Society of Japan, Tokyo (2006)

9. Błocki, Z.: Suita conjecture and the Ohsawa-Takegoshi extension theorem. Invent. Math. 193(1), 149-158 (2013)

10. Błocki, Z.: Bergman kernel and pluripotential theory. In: Proceedings of the Conference in Honor of Duong Phong. Contemporary Mathematics. American Mathematical Society (2014, to appear)

11. Błocki, Z.: Cauchy-Riemann meet Monge-Ampére. Bull. Math. Sci. 4, 433-480 (2014)

12. Błocki, Z., Pflug, P.: Hyperconvexity and Bergman completeness. Nagoya Math. J. 151, 221-225 (1998)

13. Błocki, Z., Zwonek, W.: Estimates for the Bergman kernel and the multidimensional Suita conjecture (2014, preprint)

14. Boas, H.P.: Counterexample to the Lu Qi-Keng conjecture. Proc. Am. Math. Soc. 97, 374-375 (1986)

15. Boas, H.P.: Lu Qi-Keng's problem. J. Korean Math. Soc. 37(2), 253-267 (2000). Several complex variables (Seoul, 1998)

16. Boas, H.P., Fu, S., Straube, E.J.: The Bergman kernel function: explicit formulas and zeroes. Proc. Am. Math. Soc. 127(3), 805-811 (1999)

17. Chakrabarti, D., Verma, K.: Condition R and proper holomorphic maps between equidimensional product domains. Adv. Math. 248, 820-842 (2013)

18. Chen, B.Y.: Completeness of the Bergman metric on non-smooth pseudoconvex domains. Ann. Pol. Math. 71(3), 241-251 (1999)

19. Chen, B.Y.: A note on Bergman completeness. Int. J. Math. 12(4), 383-392 (2001)

20. Christ, M.: Global $\mathcal{C}^{\infty}$-irregularity of the $\bar{\partial}$-Neumann problem for worm domains. J. Am. Math. Soc. 9(4), 1171-1185 (1996)

21. Conway, J.B.: Functions of One Complex Variable. II. Springer, New York (1995)

22. Diederich, K., Fornaess, J.E.: Boundary regularity of proper holomorphic mappings. Invent. Math. 67(3), 363-384 (1982) 
23. Diederich, K., Ohsawa, T.: An estimate for the Bergman distance on pseudoconvex domains. Ann. Math. (2) 141(1), 181-190 (1995)

24. Fefferman, C.: The Bergman kernel and biholomorphic mappings of pseudoconvex domains. Invent. Math. 26, 1-65 (1974)

25. Herbig, A.-K., McNeal, J.D., Straube, E.J.: Duality of holomorphic function spaces and smoothing properties of the Bergman projection. Trans. Am. Math. Soc. 366(2), 647-665 (2014)

26. Herbort, G.: The Bergman metric on hyperconvex domains. Math. Z. 232, 183-196 (1999)

27. Jarnicki, M., Pflug, P.: Extension of Holomorphic Functions. Walter de Gruyter, Berlin/New York (2000)

28. Jarnicki, M., Pflug, P.: Invariant Distances and Metrics in Complex Analysis, 2nd extended edn. Walter de Gruyter, Berlin (2013)

29. Josefson, B.: On the equivalence between locally polar and globally polar sets for plurisubharmonic functions on $\mathbb{C}^{n}$. Ark. Mat. 16(1), 109-115 (1978)

30. Jucha, P.: Bergman completeness of Zalcman type domains. Studia Math. 163(1), 71-83 (2004)

31. Jucha, P.: A remark on the dimension of the Bergman space of some Hartogs domains. J. Geom. Anal. 22(1), 23-37 (2012)

32. Klimek, M.: Extremal plurisubharmonic functions and invariant pseudodistances. Bull. Soc. Math. Fr. 113(2), 231-240 (1985)

33. Klimek, M.: Pluripotential Theory, Clarendon Press, Oxford/New York (1991)

34. Kobayashi, S.: Geometry of bounded domains. Trans. Am. Math. Soc. 92, 267-290 (1959)

35. Kosiński, Ł.: Geometry of quasi-circular domains and applications to tetrablock. Proc. Am. Math. Soc. 139(2), 559-569 (2011)

36. Lempert, L.: La métrique de Kobayashi et la représentation des domaines sur la boule. Bull. Soc. Math. Fr. 109, 427-474 (1981)

37. Qi-Keng, L.: On Kaehler manifolds with constant curvature. Chin. Math. Acta 8, 283-298 (1966)

38. Ohsawa, T.: Addendum to "On the Bergman kernel of hyperconvex domains". Nagoya Math. J. 137, 145-148 (1995)

39. Ohsawa, T., Takegoshi, K.: On the extension of $\mathcal{L}^{2}$ holomorphic functions. Math. Z. 195(2), 197-204 (1987)

40. Pflug, P., Zwonek, W.: $L_{h}^{2}$-domains of holomorphy and the Bergman kernel. Studia Math. 151(2), 99-108 (2002)

41. Pflug, P., Zwonek, W.: Logarithmic capacity and Bergman functions. Arch. Math. 80, 536-552 (2003)

42. Ransford, T.: Potential Theory in the Complex Plane. London Mathematical Society Student Texts, vol. 28. Cambridge University Press, Cambridge (1995)

43. Rosenthal, P.: On the zeros of the Bergman function in double-connected domains. Proc. Am. Math. Soc. 21, 33-35 (1969)

44. Skwarczyński, M.: Biholomorphic invariants related to the Bergman function. Diss. Math. 173, 1-59 (1980)

45. Suita, N.: Capacities and kernels on Riemann surfaces. Arch. Ration. Mech. Anal. 46, 212-217 (1972)

46. Suita, N., Yamada, A.: On the Lu Qi-Keng conjecture. Proc. Am. Math. Soc. 59, 222-224 (1976)

47. Szafraniec, F.H.: The reproducing kernel property and its space: the basics. In: Alpay, D. (ed.) Operator Theory, chapter 1, pp. 3-30, Springer, Basel (2015). doi:10.1007/978-3-0348-0692-3_65

48. Wiegerinck, J.: Domains with finite dimensional Bergman space. Math. Z. 187, 559-562 (1984)

49. Zwonek, W.: Regularity properties of the Azukawa metric. J. Math. Soc. Jpn. 52(4), 899-914 (2000)

50. Zwonek, W.: An example concerning Bergman completeness. Nagoya Math. J. 164, 89-101 (2001)

51. Zwonek, W.: Wiener's type criterion for Bergman exhaustiveness. Bull. Pol. Acad. Sci. Math. 50(3), 297-311 (2002) 